\title{
A Handbook of Statistical Analyses Using R
}

Brian S. Everitt and Torsten Hothorn 


\section{Logistic Regression and Generalised Linear Models: Blood Screening, Women's Role in Society, and Colonic Polyps}

\subsection{Introduction}

6.2 Logistic Regression and Generalised Linear Models

\subsection{Analysis Using R}

\subsubsection{ESR and Plasma Proteins}

We can now fit a logistic regression model to the data using the glm function. We start with a model that includes only a single explanatory variable, fibrinogen. The code to fit the model is R> plasma_glm_1 <- glm(ESR fibrinogen, data = plasma, $+\quad$ family = binomial ())

The formula implicitly defines a parameter for the global mean (the intercept term) as discussed in Chapters ?? and ??. The distribution of the response is defined by the family argument, a binomial distribution in our case. (The default link function when the binomial family is requested is the logistic function.)

From the results in Figure 6.2 we see that the regression coefficient for fibrinogen is significant at the $5 \%$ level. An increase of one unit in this variable increases the log-odds in favour of an ESR value greater than 20 by an estimated 1.83 with $95 \%$ confidence interval

$\mathrm{R}>$ confint (plasma_glm_1, parm = "fibrinogen")

$$
2.5 \div 97.5 \div
$$

0.33876193 .9984921

These values are more helpful if converted to the corresponding values for the odds themselves by exponentiating the estimate

$R>\exp ($ coef (plasma_glm_1) ["fibrinogen"])

$$
\begin{array}{r}
\text { fibrinogen } \\
6.215715
\end{array}
$$

and the confidence interval

$\mathrm{R}>\exp (\operatorname{confint}($ plasma_glm_1, parm = "fibrinogen")) 

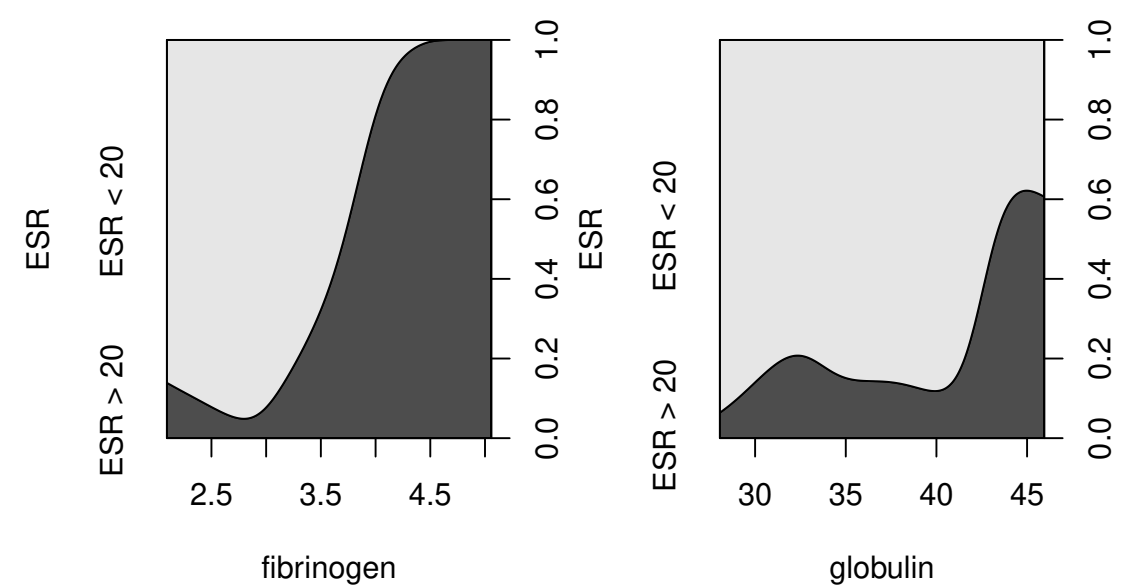

Figure 6.1 Conditional density plots of the erythrocyte sedimentation rate (ESR) given fibrinogen and globulin.

$$
\begin{array}{rr}
2.5 \% & 97.5 \% \\
1.403209 & 54.515884
\end{array}
$$

The confidence interval is very wide because there are few observations overall and very few where the ESR value is greater than 20. Nevertheless it seems likely that increased values of fibrinogen lead to a greater probability of an ESR value greater than 20 .

We can now fit a logistic regression model that includes both explanatory variables using the code

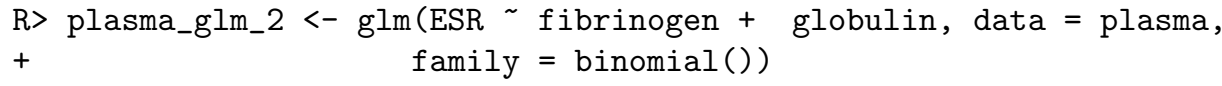

and the output of the summary method is shown in Figure 6.3.

The coefficient for gamma globulin is not significantly different from zero. Subtracting the residual deviance of the second model from the corresponding value for the first model we get a value of 1.87. Tested using a $\chi^{2}$-distribution with a single degree of freedom this is not significant at the $5 \%$ level and so we conclude that gamma globulin is not associated with ESR level. In R, the task of comparing the two nested models can be performed using the anova function 


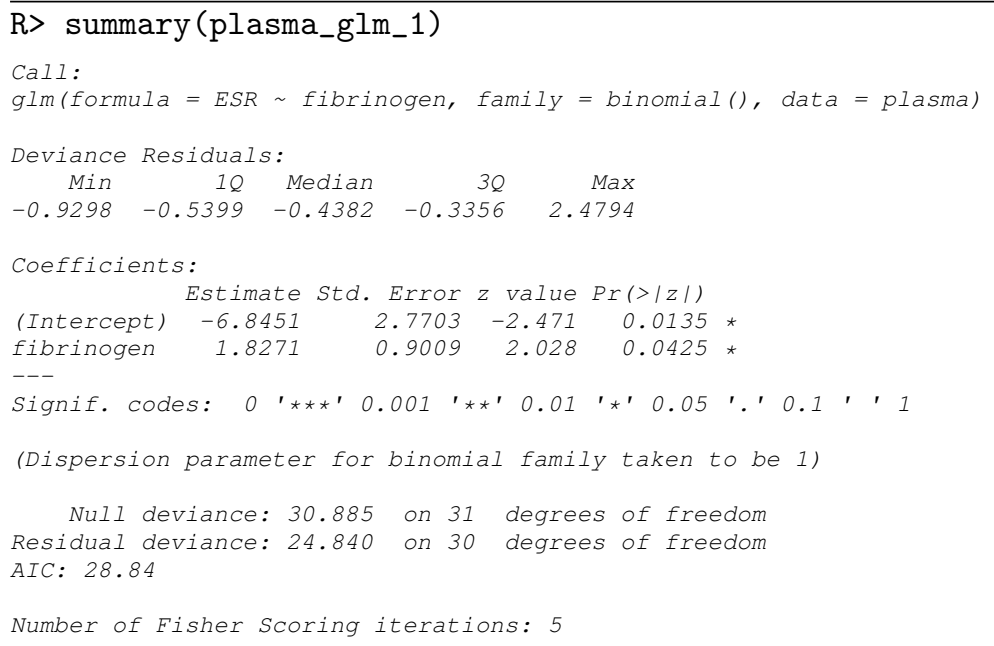

Figure 6.2 R output of the summary method for the logistic regression model fitted to the plasma data.

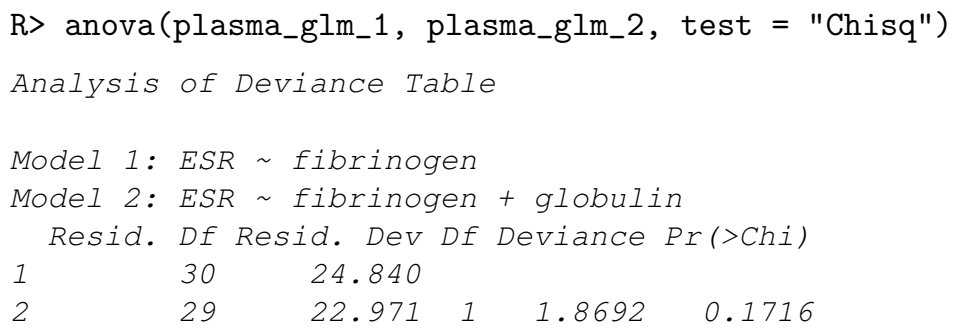

Nevertheless we shall use the predicted values from the second model and plot them against the values of both explanatory variables using a bubble plot to illustrate the use of the symbols function. The estimated conditional probability of a ESR value larger 20 for all observations can be computed, following formula (??), by

R> prob <- predict (plasma_glm_2, type = "response")

and now we can assign a larger circle to observations with larger probability as shown in Figure 6.4. The plot clearly shows the increasing probability of an ESR value above 20 (larger circles) as the values of fibrinogen, and to a lesser extent, gamma globulin, increase.

\subsubsection{Women's Role in Society}

Originally the data in Table ?? would have been in a completely equivalent form to the data in Table ?? data, but here the individual observations have been grouped into counts of numbers of agreements and disagreements for 


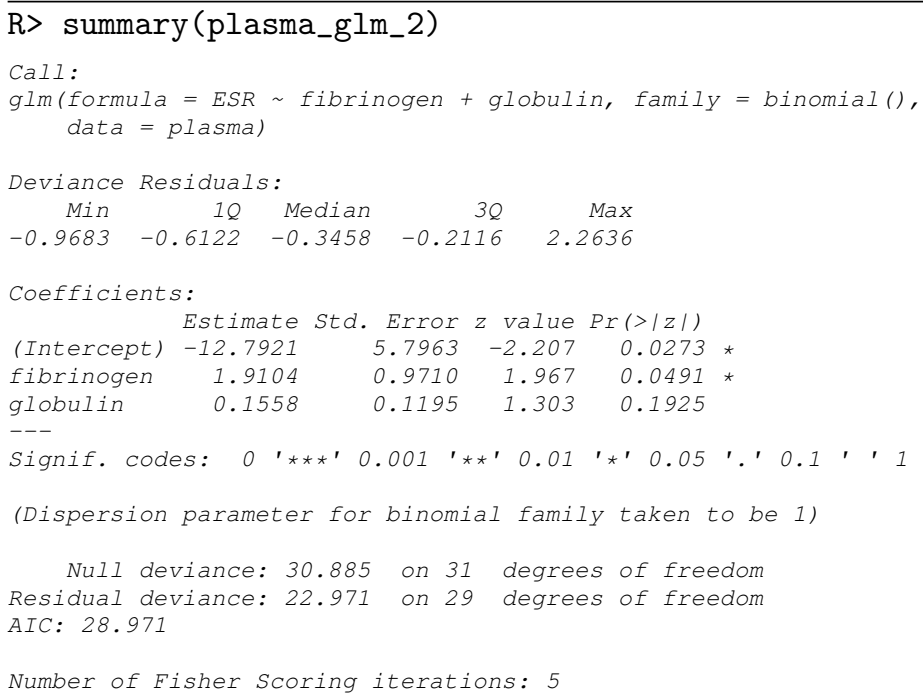

Figure 6.3 R output of the summary method for the logistic regression model fitted to the plasma data.

the two explanatory variables, sex and education. To fit a logistic regression model to such grouped data using the glm function we need to specify the number of agreements and disagreements as a two-column matrix on the left hand side of the model formula. We first fit a model that includes the two explanatory variables using the code

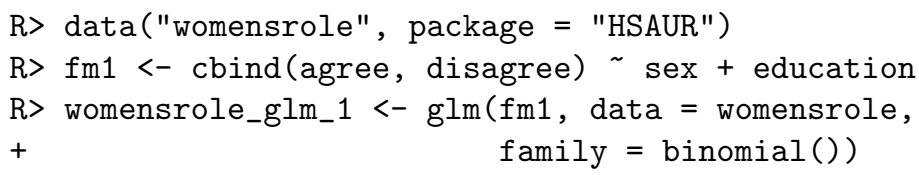

From the summary output in Figure 6.5 it appears that education has a highly significant part to play in predicting whether a respondent will agree with the statement read to them, but the respondent's sex is apparently unimportant. As years of education increase the probability of agreeing with the statement declines. We now are going to construct a plot comparing the observed proportions of agreeing with those fitted by our fitted model. Because we will reuse this plot for another fitted object later on, we define a function which plots years of education against some fitted probabilities, e.g.,

$\mathrm{R}>$ role.fitted1 <- predict(womensrole_glm_1, type = "response") and labels each observation with the person's sex:

$\mathrm{R}>$ myplot <- function(role.fitted) \{

$+\quad f<-$ womensrole\$sex == "Female"

$+\quad$ plot (womensrole\$education, role.fitted, type = "n",

$+\quad \mathrm{ylab}=$ "Probability of agreeing", 
ANALYSIS USING R

R> plot(globulin fibrinogen, data $=$ plasma, $x \lim =\mathrm{c}(2,6)$, $+\quad$ ylim $=c(25,55)$, pch $=" . ")$

R> symbols (plasma\$fibrinogen, plasma\$globulin, circles = prob,

$+\quad$ add $=$ TRUE)

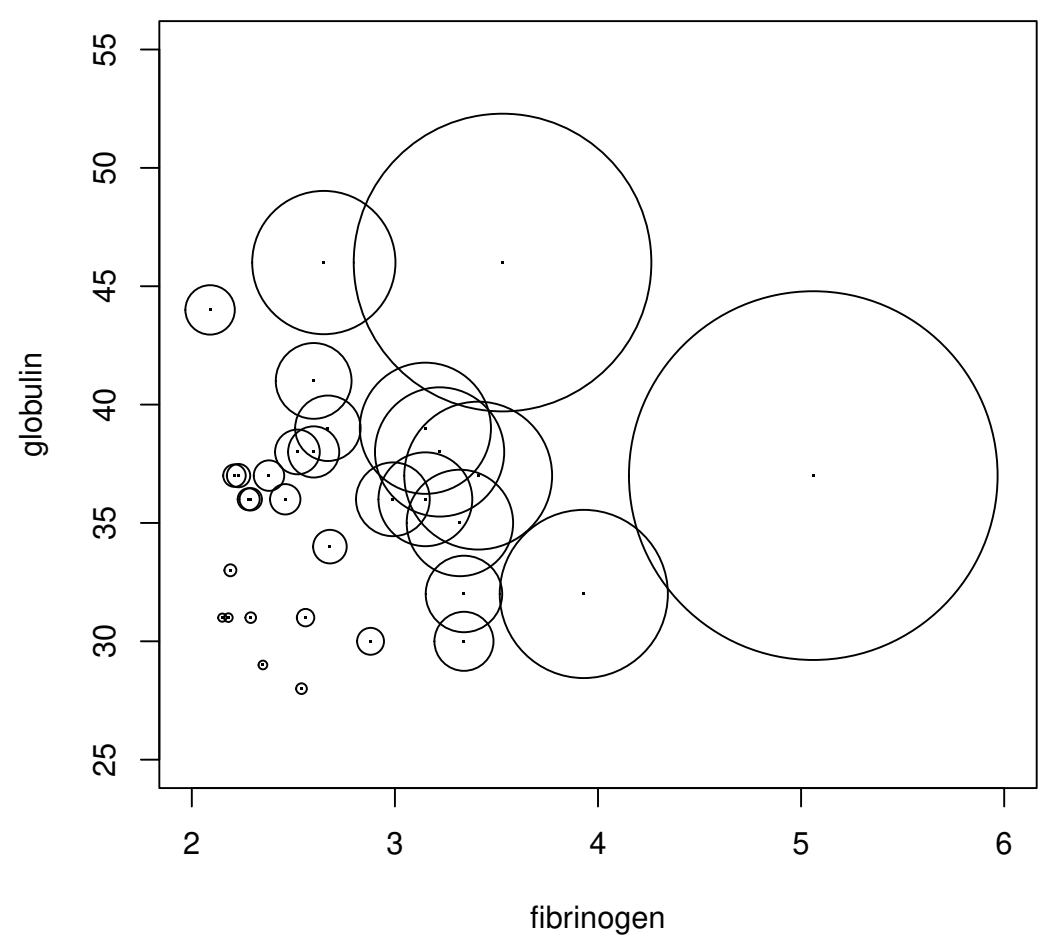

Figure 6.4 Bubble plot of fitted values for a logistic regression model fitted to the ESR data.

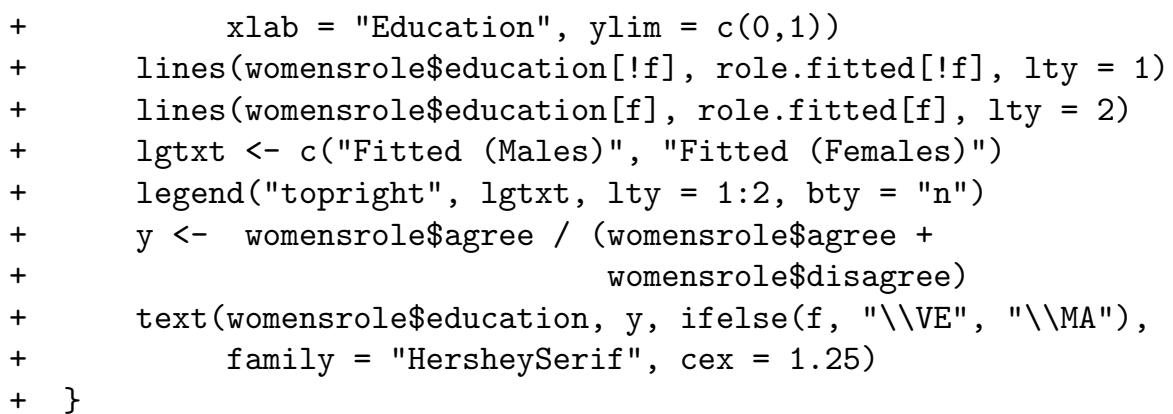




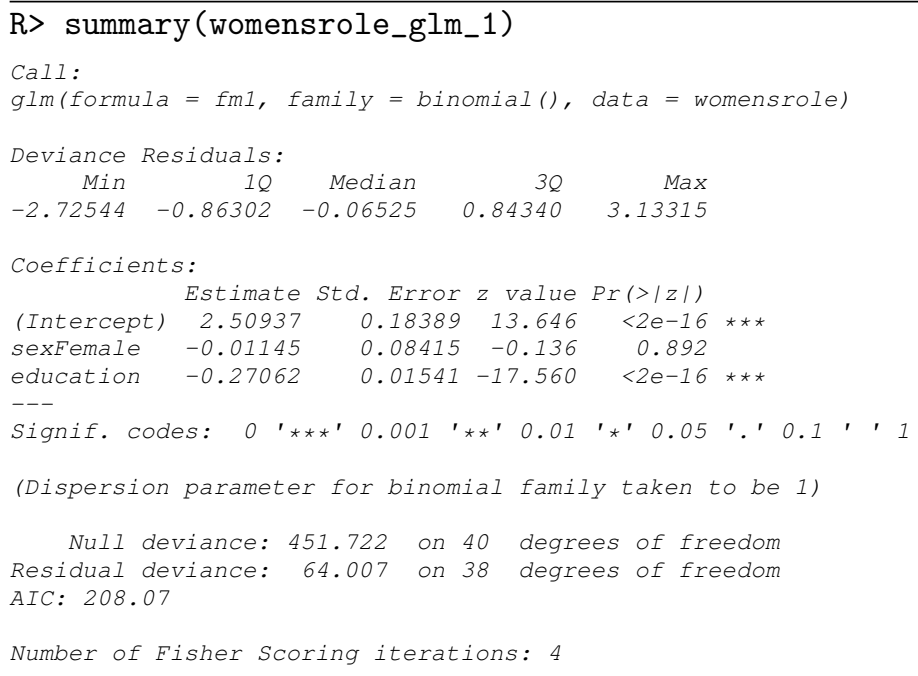

Figure 6.5 R output of the summary method for the logistic regression model fitted to the womensrole data.

The two curves for males and females in Figure 6.6 are almost the same reflecting the non-significant value of the regression coefficient for sex in womensrole_glm_1. But the observed values plotted on Figure 6.6 suggest that there might be an interaction of education and sex, a possibility that can be investigated by applying a further logistic regression model using

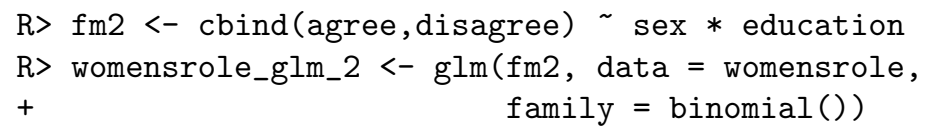

The sex and education interaction term is seen to be highly significant, as can be seen from the summary output in Figure 6.7.

We can obtain a plot of deviance residuals plotted against fitted values using the following code above Figure 6.9. The residuals fall into a horizontal band between -2 and 2 . This pattern does not suggest a poor fit for any particular observation or subset of observations.

\subsubsection{Colonic Polyps}

The data on colonic polyps in Table ?? involves count data. We could try to model this using multiple regression but there are two problems. The first is that a response that is a count can only take positive values, and secondly such a variable is unlikely to have a normal distribution. Instead we will apply a GLM with a log link function, ensuring that fitted values are positive, and 
R> myplot (role.fitted1)

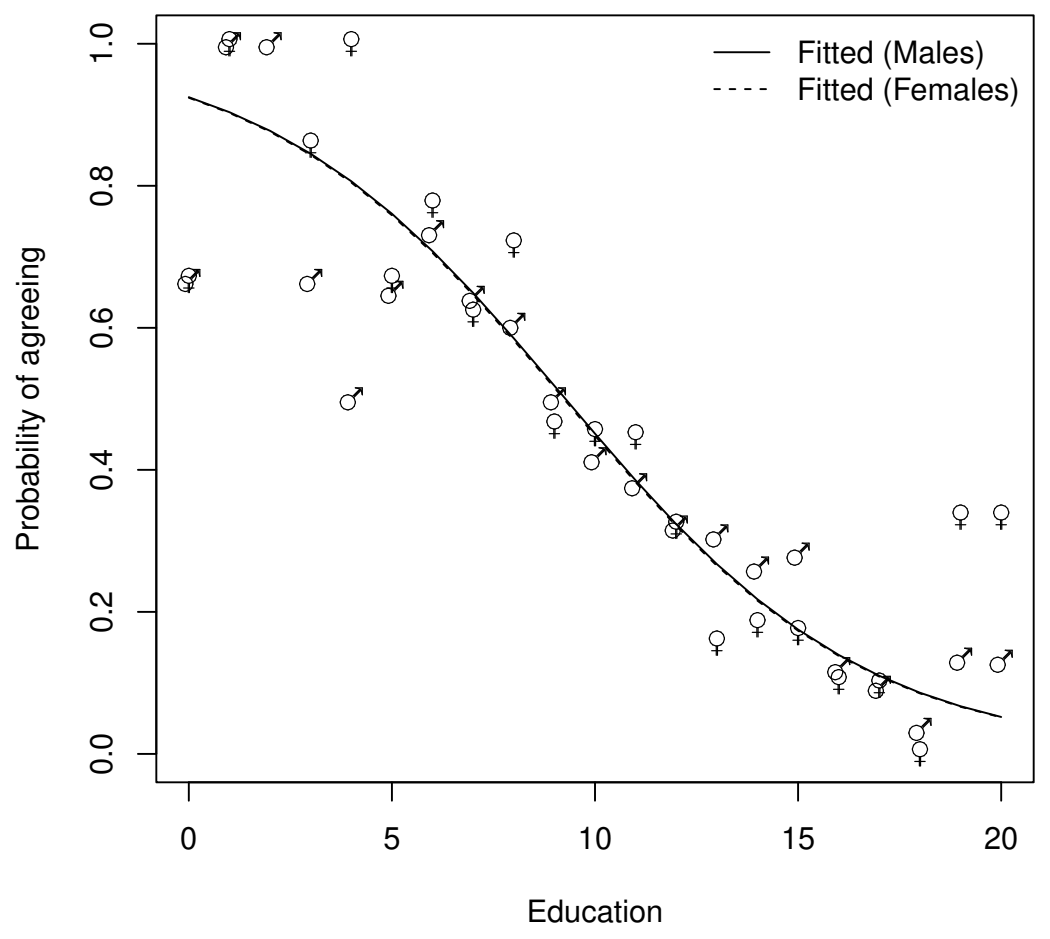

Figure 6.6 Fitted (from womensrole_glm_1) and observed probabilities of agreeing for the womensrole data.

a Poisson error distribution, i.e.,

$$
\mathrm{P}(y)=\frac{e^{-\lambda} \lambda^{y}}{y !} .
$$

This type of GLM is often known as Poisson regression. We can apply the model using

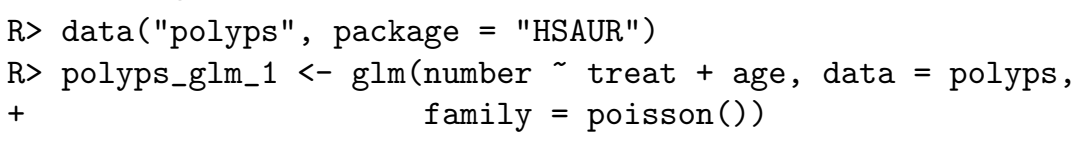

(The default link function when the Poisson family is requested is the log function.)

We can deal with overdispersion by using a procedure known as quasilikelihood, which allows the estimation of model parameters without fully 


\section{LOGISTIC REGRESSION AND GENERALISED LINEAR MODELS}

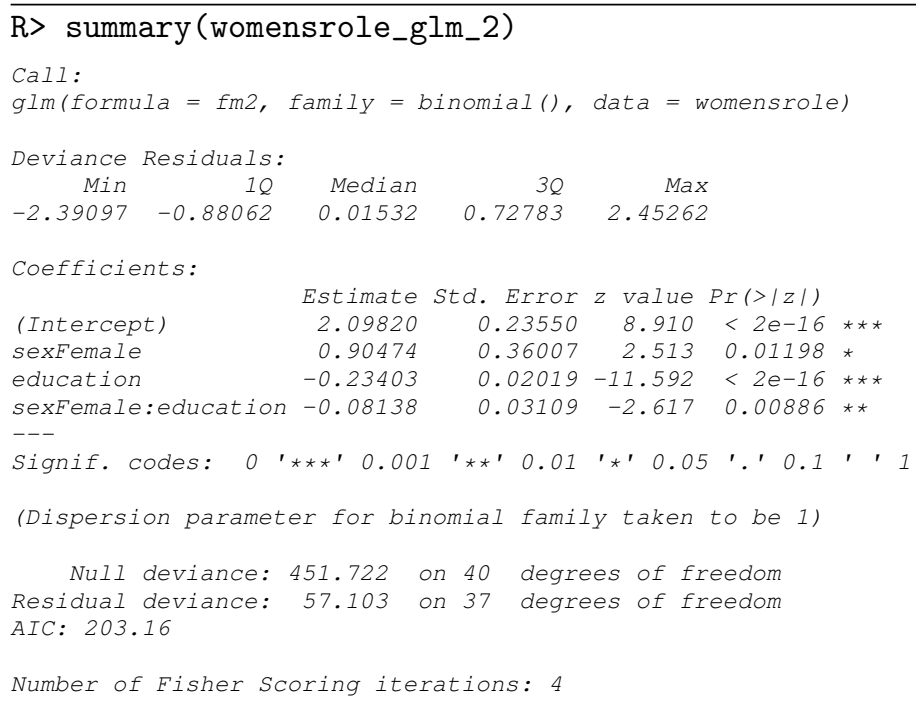

Figure 6.7 $\mathrm{R}$ output of the summary method for the logistic regression model fitted to the womensrole data.

knowing the error distribution of the response variable. McCullagh and Nelder (1989) give full details of the quasi-likelihood approach. In many respects it simply allows for the estimation of $\phi$ from the data rather than defining it to be unity for the binomial and Poisson distributions. We can apply quasilikelihood estimation to the colonic polyps data using the following $\mathrm{R}$ code

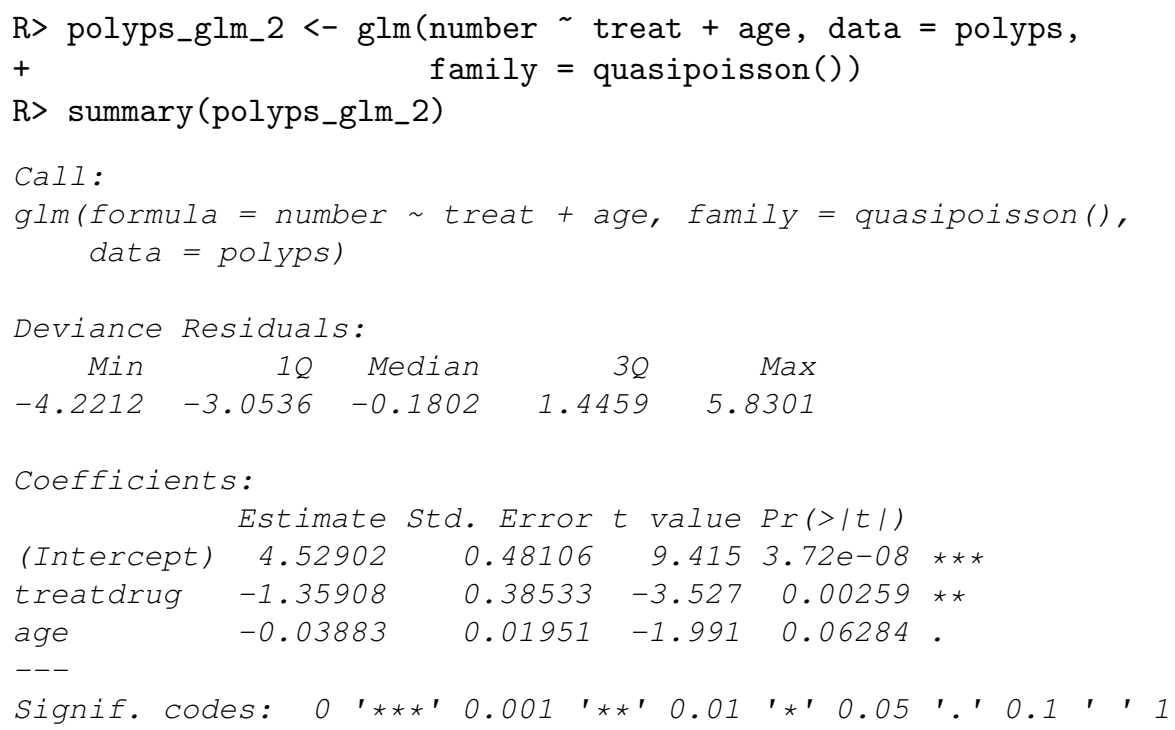


$\mathrm{R}>$ role.fitted2 <- predict (womensrole_glm_2, type = "response")

R> myplot (role.fitted2)

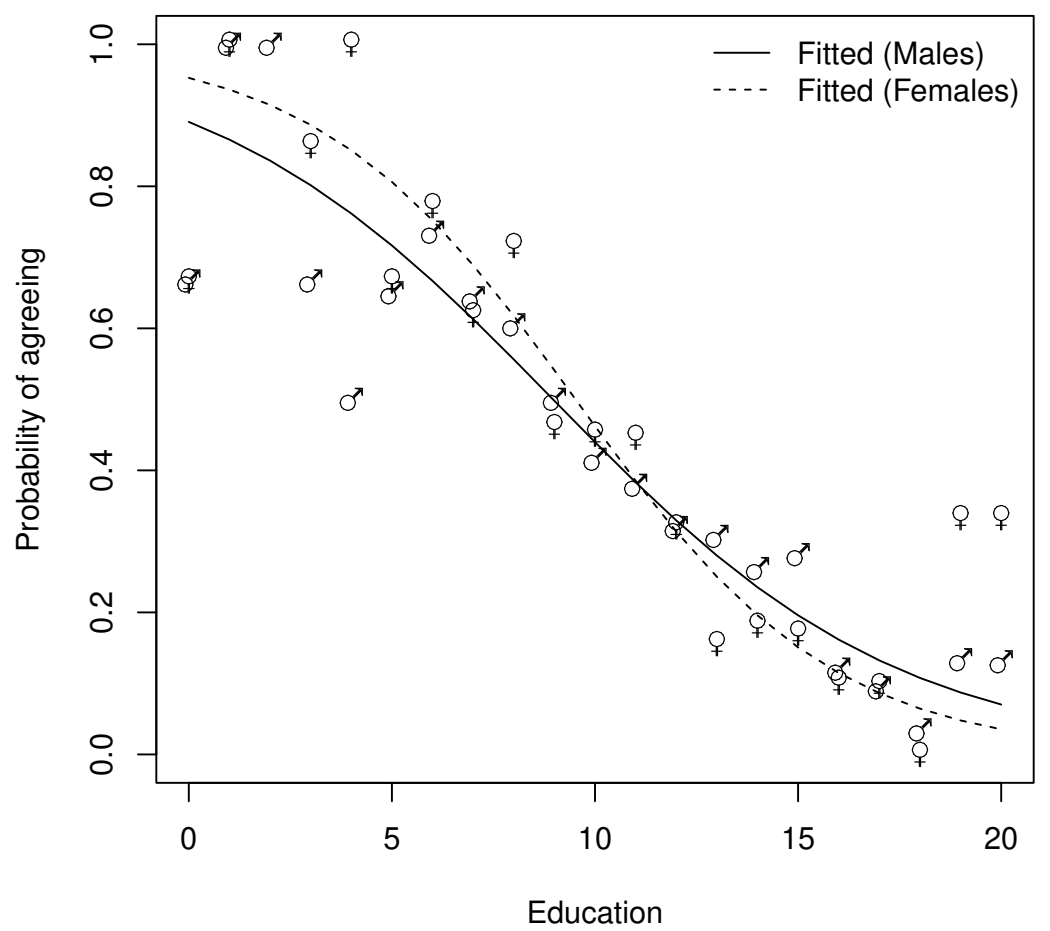

Figure 6.8 Fitted (from womensrole_glm_2) and observed probabilities of agreeing for the womensrole data.

(Dispersion parameter for quasipoisson family taken to be 10.72805)

Null deviance: 378.66 on 19 degrees of freedom

Residual deviance: 179.54 on 17 degrees of freedom AIC: NA

Number of Fisher Scoring iterations: 5

The regression coefficients for both explanatory variables remain significant but their estimated standard errors are now much greater than the values given in Figure 6.10. A possible reason for overdispersion in these data is that polyps do not occur independently of one another, but instead may 'cluster' together. 

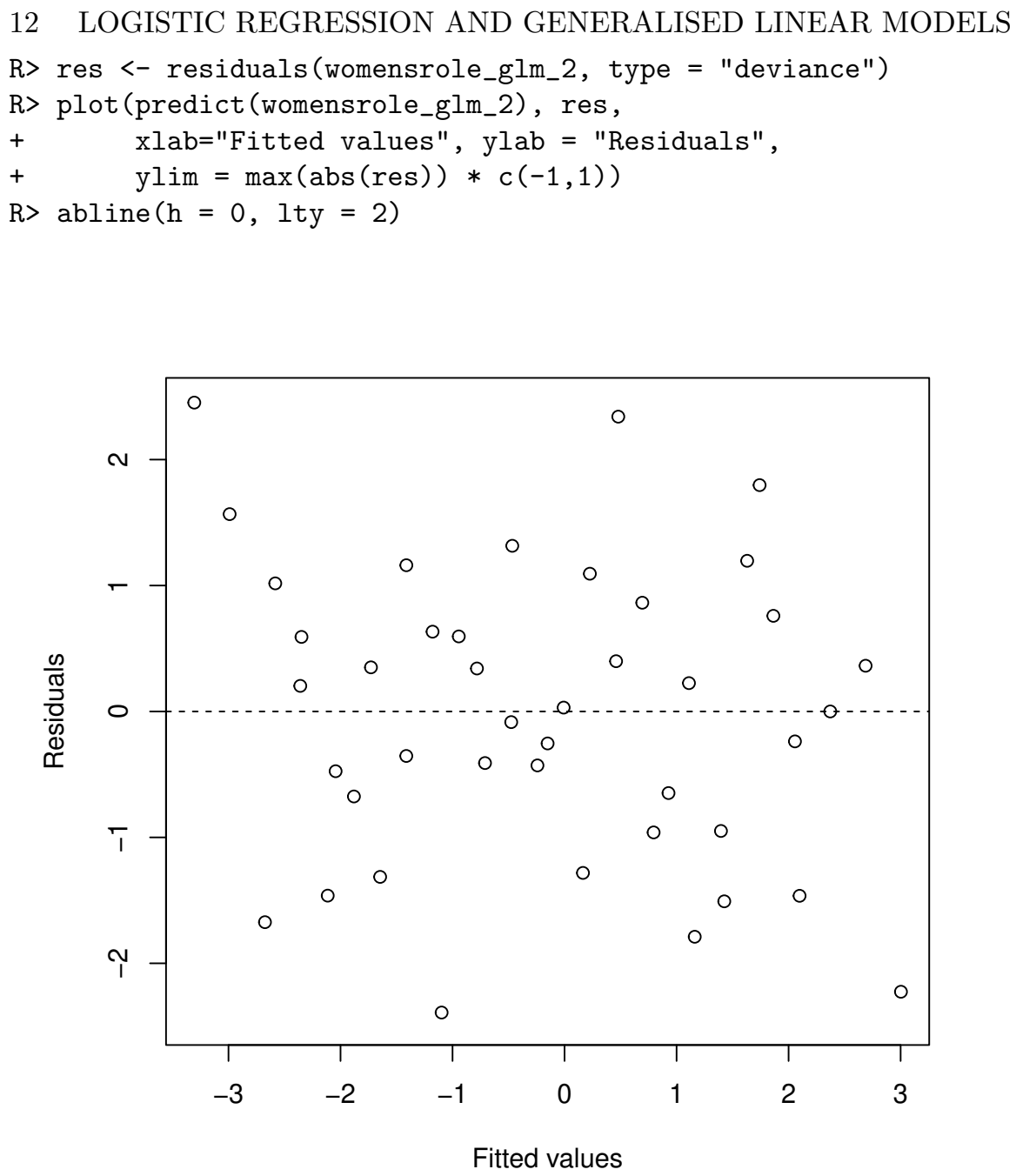

Figure 6.9 Plot of deviance residuals from logistic regression model fitted to the womensrole data. 


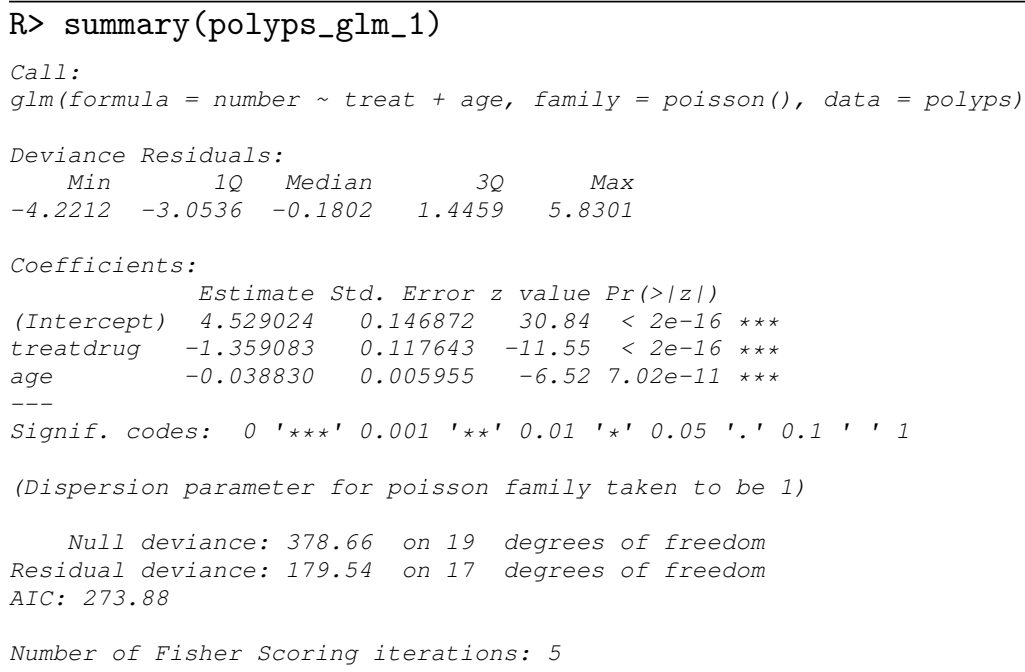

Figure 6.10 R output of the summary method for the Poisson regression model fitted to the polyps data. 


\section{Bibliography}

McCullagh, P. and Nelder, J. A. (1989), Generalized Linear Models, London, UK: Chapman \& Hall/CRC. 